\title{
CONTEXTUALIZACIÓN DEL INICIO SEXUAL Y BARRERAS INDIVIDUALES DEL USO DE ANTICONCEPTIVOS EN ADOLESCENTES DE LIMA, HUAMANGA E IQUITOS
}

\author{
Gloria Carmona ${ }^{1, a}$, Jessica Beltran²,b, María Calderón ${ }^{3, c}$, Marina Piazza $^{1,5, d}$, Susana Chávez ${ }^{4, e}$
}

\begin{abstract}
RESUMEN
Objetivos. Contextualizar el inicio sexual y las barreras individuales hacia el uso de anticonceptivos en adolescentes, con conocimiento adecuados sobre anticoncepción y enfermedades de transmisión sexual. Materiales y métodos. Se desarrolló un estudio cuanticualitativo en adolescentes procedentes de ciudades con altas tasas de embarazo: Iquitos, Huamanga y Lima. La convocatoria se realizó a través de Facebook, luego se aplicaron encuestas para seleccionar a los adolescentes que cumplían los criterios de inclusión y, finalmente, se realizaron entrevistas para abordar el tema en profundidad. Resultados. Participaron 33 mujeres y 23 varones adolescentes urbanos, con edades entre 18 y 19 años. La edad de inicio sexual osciló entre los 14 y 16 años; las principales razones de inicio sexual en los varones fue el deseo sexual; en las mujeres fue la presión de la pareja; la persona con la que se iniciaron fue, principalmente, una pareja ocasional en los varones, y en las mujeres fue "su enamorado". Se identificaron barreras individuales en relación al uso de anticonceptivos como la estabilidad de la pareja, la responsabilidad de la mujer al usar anticonceptivos, la limitada capacidad de negociación y temor al abandono del varón por exigir su uso, además de la reducción de la sensibilidad sexual generada por el uso del condón. Conclusiones. Estas barreras limitan el uso de anticonceptivos, sugiriendo la necesidad de desarrollar estrategias específicas para fortalecer las habilidades interpersonales del adolescente que se extienden más allá de ofrecer información acerca de los riesgos del sexo sin protección.
\end{abstract}

Palabras clave: Adolescentes; Anticonceptivos; Género; Conducta sexual; Medios de comunicación social (fuente: DeCS BIREME).

\section{CONTEXTUALIZATION OF SEXUAL INITIATION AND INDIVIDUAL BARRIERS TO THE USE OF CONTRACEPTIVES BY ADOLESCENTS OF LIMA, HUAMANGA, AND IQUITOS}

\begin{abstract}
Objectives. To contextualize sexual initiation and barriers to the use of contraceptives by adolescents as well as the acquisition of adequate knowledge regarding contraception and sexually transmitted diseases. Materials and methods. This quantitative and qualitative study evaluated adolescents living in three cities with high pregnancy rates in Peru: lquitos, Huamanga, and Lima. Subjects were invited to participate in the study via Facebook. Questionnaires were then used to select adolescents who met the inclusion criteria, and interviews were conducted to obtain detailed data regarding the study topic. Results. A total of 33 women and 23 men aged 18 to 19 years participated in the study. The age of onset of sexual activity ranged from 14 to 16 years; the main reasons for sexual initiation were sexual desire among men and pressure from the partner among women; in most cases, the sexual partner was an occasional companion for men and a boyfriend for women. The barriers related to the use of contraceptives were the financial stability of the partner, responsibility of the female partner when using contraceptives, limited capacity to negotiate choices, fear of abandonment by the male partner for demanding the use of contraceptives, and the reduction of sexual sensitivity with the use of condoms. Conclusions. The barriers identified in this study limit the use of contraceptives and indicate the need to develop specific strategies to strengthen the interpersonal skills of adolescents and provide accurate information about the risks of unprotected sex.
\end{abstract}

Keywords: Adolescents; Contraceptives; Gender; Sexual behavior; Social media (source: MeSH NLM).

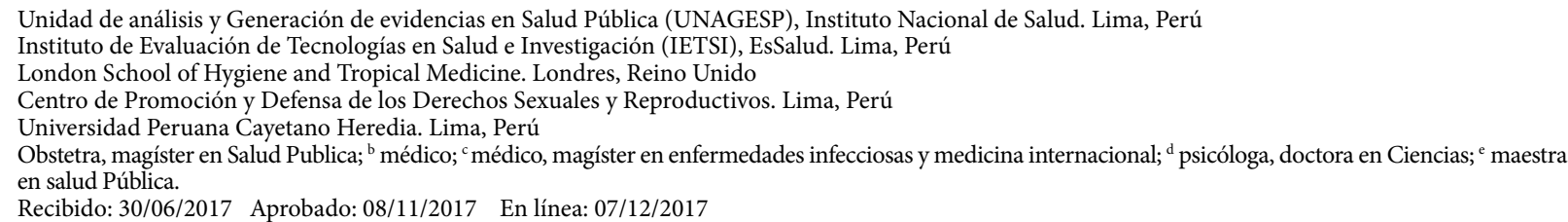

Citar como: Carmona G, Beltran J, Calderón M, Piazza M, Chávez S. Contextualización del inicio sexual y barreras individuales del uso de anticonceptivos en adolescentes de Lima, Huamanga e Iquitos. Rev Peru Med Exp Salud Publica. 2017;34(4):601-10. doi: 10.17843/rpmesp.2017.344.2971 


\section{INTRODUCCIÓN}

Los adolescentes cumplen un rol fundamental en el desarrollo de los países en el corto y mediano plazo, por ser los hacedores mediatos del futuro del país tanto en su aspecto económico como social; sentando las bases para la siguiente generación y modificando la sociedad que hayan recibido. Por ello, alcanzar su desarrollo integral con una perspectiva de equidad de género, forma parte de los objetivos de desarrollo sostenible aprobados por los estados miembros de las Naciones Unidas ${ }^{(1)}$. Dentro de este desarrollo integral, la salud sexual y reproductiva tiene un rol fundamental en su desarrollo y proyecto de vida personal. En el Perú, el embarazo adolescente (EA) se presenta en $14,6 \%$ de esta población y entre los departamentos con las tasas más altas encontramos a Loreto, Ayacucho y Lima con 10,6\%, 22,3\% y 25\%, respectivamente ${ }^{(2)}$. Asimismo, entre los adolescentes la prevalencia del virus de inmunodeficiencia humana (VIH), llegó a 287 casos confirmados y notificados desde 1983 hasta mediados del $2017^{(3)}$. Ambas situaciones afectan el desarrollo y proyecto de vida de los adolescentes, e impactan en su entorno social y en el de su comunidad.

En Latinoamérica y el Caribe, la edad promedio del inicio sexual es menor en varones que en mujeres, los rangos fluctúan desde 12,7 a 16 años en varones y desde 15,6 hasta 17,9 años en mujeres. Por otro lado, a pesar de que la mayoría de los adolescentes tienen conocimientos sobre anticoncepción, el uso de estos métodos aún es bajo ${ }^{(4)}$. En el Perú, la Encuesta Demográfica y de Salud Familiar (ENDES) del 2014 encontró que el 25\% de mujeres entre los 15 a 19 años de edad mantenían una vida sexual activa y habían tenido más de una pareja sexual ${ }^{(2)}$. Un estudio en escolares de secundaria de Perú, evidenció que el $11 \%$ de los adolescentes mantiene una vida sexual activa, principalmente los varones. El informe revela que el $37,1 \%$ utiliza, a veces, algún método anticonceptivo y que el $29,2 \%$, no utiliza ningún método durante sus relaciones sexuales ${ }^{(5)}$

En respuesta a esta situación, instituciones de múltiples sectores, públicas como privadas, han generado intervenciones educativas, promocionales o preventivas; algunas de ellas con un enfoque según género y con disponibilidad a mejorar sus indicadores y estrategias de efectividad, considerando cada vez mayor atención a las evidencias científicas reportadas ${ }^{(6,7)}$. Por otro lado, a pesar de que no existe consenso en la literatura científica respecto al nivel de información que manejan los adolescentes acerca de su salud sexual y reproductiva ${ }^{(8,9)}$, sí existe consenso acerca de que los adolescentes no usan anticonceptivos de manera suficiente ${ }^{(4,8,9)}$. Esto indica que la información no se traduce, necesariamente, en una conducta preventiva.

\section{MENSAJES CLAVE}

Motivación para realizar el estudio. En el Perú, los embarazos no deseados, las enfermedades de transmisión sexual y los abortos subreportados e inseguros en adolescentes tienen una alta frecuencia, existe limitada evidencia de que las intervenciones informativas funcionen, por lo que urge identificar cuáles son las barreras adicionales que influyen en que el adolescente que lo requiera, utilice un anticonceptivo.

Principales hallazgos. Las barreras identificadas fueron: la limitada capacidad de negociación, la estabilidad de la pareja, la responsabilidad del uso de anticonceptivos atribuida a la mujer y su temor por ser abandonada por el varón en caso de exigir uso de anticonceptivos.

Implicancias. Intervenciones que además de brindar información sobre anticonceptivos incluyan habilidades interpersonales con enfoque de género podrían optimizar la conducta sexual preventiva en los adolescentes.

Evidencia desarrollada en Perú, reporta que son las cuestiones de vergüenza e incomodidad las razones por las cuales los adolescentes no usan condones, y no su costo económico ${ }^{(10,11)}$. Sin embargo, el significado social de ser varón o mujer relacionada con la capacidad de negociación, el manejo de su sexualidad, de la asertividad, entre otros; son factores que requieren ser más explorados en relación al uso de anticonceptivos.

En ese sentido, el objetivo de este estudio fue contextualizar el inicio sexual e identificar las barreras individuales hacia el uso de anticonceptivos en aquellos adolescentes que, a pesar de tener el conocimiento sobre anticoncepción y enfermedades de transmisión sexual (ETS), no utilizan anticonceptivos. Estos hallazgos podrían colaborar en la identificación de oportunidades de acción que podrían ser consideradas o complementadas en las actuales intervenciones de salud sexual, para que, además de brindar información, también se fortalezcan diversos factores subjetivos como el género, la capacidad de negociación, entre otros, que interfieren en el acto de ejercer una conducta preventiva.

\section{MATERIALES Y MÉTODOS}

\section{TIPO DE ESTUDIO}

El presente estudio tiene un diseño mixto secuencial utilizando, en primer lugar, un componente cuantitativo, a través de una encuesta para seleccionar a los participantes $y$, en segundo lugar, se empleó un componente cualitativo a través de una entrevista semiestructurada.

\section{LUGAR DEL ESTUDIO}

El ámbito geográfico del estudio incluye Lima, Ayacucho y Loreto, por ser algunos de los departamentos con mayores 
tasas de EA según los últimos reportes nacionales ${ }^{(2)}$. Participaron estudiantes de la Universidad de Ayacucho Federico Froebel, de la Universidad Nacional de la Amazonía Peruana de Loreto y usuarios del Centro de Salud Mi Perú de Lima. Asimismo, se incluyeron a adolescentes de la jurisdicción que voluntariamente manifestaron su deseo de participar en el estudio.

\section{SELECCIÓN DE PARTICIPANTES}

Se incluyeron adolescentes urbanos de 18 y 19 años que, a pesar de tener conocimientos adecuados sobre anticoncepción y $\mathrm{VIH}$, manifestaron no utilizar anticonceptivos en sus relaciones sexuales. Para poder identificar a los adolescentes con este perfil, se realizó una convocatoria a través de Facebook y luego se utilizó una encuesta validada, donde se identificaron a los adolescentes con la variable de selección.

\section{VARIABLES}

Se definió como conocimientos adecuados sobre VIH y métodos anticonceptivos a aquellos adolescentes que contestaron asertivamente más del $50 \%$ de las preguntas de la encuesta virtual o física. El no uso de anticonceptivos fue la variable de selección, y se definió como aquellos que respondieron negativamente a las preguntas referentes al uso de anticonceptivos. Las caracteristicas del inicio sexual y las barreras individuales fueron identificadas en base a los datos brindados en la entrevistas semiestructuras.

\section{INSTRUMENTOS}

El instrumento utilizado se basó en dos encuestas validadas ${ }^{(12,13)}$ y estuvo conformado por 14 preguntas, de las cuales 10 fueron de tipo general y estuvieron dirigidas a ambos sexos (seis sobre conocimientos de VIH y cuatro sobre conocimientos de métodos anticonceptivos) y cuatro preguntas sobre el uso de anticonceptivos según sexo (por ejemplo, el condón en varones y el método del ritmo o píldoras en mujeres).

Para evaluar la claridad, la forma de presentación y el grado de representatividad de los instrumentos (la encuesta y la entrevista semiestructurada) se realizaron juicios por expertos y pruebas piloto, en los cuales participaron adolescentes no incluidos en la muestra, líderes de las instituciones seleccionadas y de organizaciones que promueven la salud sexual y reproductiva. Estas instituciones fueron PROMSEX (promoción de los derechos sexuales y reproductivos); UNFPA (Fondo Nacional de la Poblaciones Unidas); Departamento de Psicología de Universidad de Ayacucho Federico Froebel, y la Estrategia de Salud Sexual y Reproductiva del Ministerio de Salud a nivel central y a nivel de la región de Loreto, a fin de colaborar con la producción del conocimiento de manera multidisciplinar.

\section{PROCESO DE RECOLECCIÓN DE DATOS}

\section{Primera parte (cuantitativa)}

La convocatoria se realizó a través de una página en Facebook creada con el nombre "Loseperonolousé" (https:// www.facebook.com/Loseperonolouse/). A través de esta, se realizó la promoción de diapositivas sobre salud sexual, seleccionando cuidadosamente el contenido, de manera que resultara atractiva para los adolescentes. Estas dispositivas, además de brindar información muy puntual, invitaban a los adolescentes a participar de una encuesta virtual. A su vez, permitía la interacción de comentarios que reforzaban el mensaje y la opción de compartir el mensaje con otros amigos de Facebook.

La encuesta, en su versión física fue distribuida en las sedes consideradas para el estudio, y en su versión virtual fue elaborada y difundida en la plataforma Google Docs (https://docs.google.com/) a través de las redes sociales. El proceso de recolección de datos con la encuesta física implicó el traslado de los investigadores a las zonas seleccionadas y tuvo una duración total de 45 días.

\section{Segunda parte (cualitativa)}

Los adolescentes seleccionados fueron invitados en forma directa o por correo electrónico a participar de una entrevista semiestructurada y retroprospectiva (conformada por preguntas abiertas sobre el presente y pasado del entrevistado en relación al tema en cuestión). Las entrevistas se realizaron en el lugar y horario de preferencia de los entrevistados, ofreciéndoles la posibilidad de acudir con algún acompañante. En promedio, las entrevistas tuvieron una duración de 45 min y fueron desarrolladas por el investigador principal o un coinvestigador, en cafés de centros comerciales, bulevares y/o en las instituciones seleccionadas, con el fin de brindarle mayor comodidad y seguridad.

En el componente cualitativo, se empleó un muestreo no probabilístico secuencial ${ }^{(14)}$ cuyo objetivo fue asegurar que la muestra fuera diversa dentro de los criterios de selección establecidos. Se realizaron el número de entrevistas necesarias hasta lograr la saturación de la información. Al final de cada entrevista se brindó a los participantes una sesión de orientación y consejería sobre algún tópico de salud sexual, según la preferencia del entrevistado. En las entrevistas se tomó como referencia el enfoque fenomenológico ${ }^{(15)}$, explorando temas emergentes preidentificados acerca de las barreras por las cuales no se utilizan anticonceptivos.

El trabajo de campo fue monitoreado y supervisado durante todo el proceso por el investigador principal, atendiendo las dudas o consultas con una retroalimentación conjunta 
del equipo de investigación, donde cada miembro tuvo un cuaderno de trabajo de campo, además de recibir una capacitación sobre los procesos y objetivos del estudio. Esta campaña duró siete fines de semana, de jueves a domingo. Es decir, que el período de reclutamiento y de entrevistas en forma virtual tuvo una duración total de 28 días.

\section{ANÁLISIS DE DATOS}

En ambos componentes, con el fin de garantizar la calidad y consistencia de la información, se realizó una limpieza de la base de datos otorgándole una identificación numérica a cada participante. El análisis fue diferenciado por género. En el componente cuantitativo, el análisis de los datos de las encuestas, se presentaron con frecuencias y porcentajes.

En el análisis de las entrevistas se empleó el modelo propuesto por Bronfenbrenner et al., que propone la comprensión de la conducta del adolescente, en función a los escenarios o sistemas en los que el adolescente se desarrolla ${ }^{(16)}$. El análisis de estos datos comprendió las siguientes fases: a) lectura y codificación de entrevistas; b) nueva lectura y codificación de entrevistas; c) identificación de regularidades y diferencias según género y contexto local, y d) elaboración de matrices de concentración de datos codificados, en relación con la teoría existente.

\section{CONSIDERACIONES ÉTICAS}

El estudio fue revisado y aprobado por el Comité de Ética e Investigación del Instituto Nacional de Salud del Perú. Se contó con consentimiento de parte de las instituciones locales, así como consentimiento escrito de los participantes.

\section{RESULTADOS}

La encuesta física fue respondida por 195 adolescentes y la encuesta virtual por 564 adolescentes. El porcentaje que cumplió con los criterios de inclusión fue de 31,7\% $(n=62)$ en la encuesta física y de $23,7 \%(n=133)$ en la encuesta virtual. Finalmente, se entrevistaron a 56 adolescentes. La Tabla 1 presenta la distribución de la muestra por sexo, lugar de procedencia, de institución y tipo de encuesta aplicada en el proceso de reclutamiento. Se realizaron, además, conversaciones informales, diversos registros durante el trabajo de campo y revisión de documentos clave. Los hallazgos de las entrevistas y las barreras individuales por las cuales los adolescentes con vida sexual activan no usan anticonceptivos, son descritos desde los sistemas o escenarios en que los adolescentes se desarrollan.

El microsistema, se refiere a las características más cercanas de la vida sexual del adolescente participante, de sus comportamientos, roles y relaciones interpersonales con la pareja sexual. Con relación a ello, en los hombres la edad de inicio sexual tiende a ser más temprana (14 o 15 años) que las mujeres (15 o 16 años). Entre los factores relacionados al inicio de la actividad sexual, las mujeres resaltan aspectos de la formación en el hogar, la comunicación, afecto, orientación y cuidado que reciben de sus padres. En contraste, los hombres refieren aspectos

Tabla 1. Características de los adolescentes según sexo y procedencia

\begin{tabular}{|c|c|c|c|c|c|c|c|c|c|}
\hline \multirow{3}{*}{ Características } & \multicolumn{4}{|c|}{ Mujeres } & \multicolumn{4}{|c|}{ Varones } & \multirow{3}{*}{ Total $(\mathbf{n}$} \\
\hline & \multicolumn{3}{|c|}{$\begin{array}{l}\text { Encuesta } \\
\text { Física }\end{array}$} & \multirow{2}{*}{$\begin{array}{c}\begin{array}{c}\text { Encuesta } \\
\text { Virtual }\end{array} \\
\text { Facebook }\end{array}$} & \multicolumn{3}{|c|}{$\begin{array}{l}\text { Encuesta } \\
\text { Física }\end{array}$} & \multirow{2}{*}{$\begin{array}{c}\text { Encuesta } \\
\text { Virtual }\end{array}$} & \\
\hline & $\begin{array}{l}\text { C.S Mi } \\
\text { Perú }\end{array}$ & UDAFF & UNAP & & $\begin{array}{l}\text { C.S Mi } \\
\text { Perú }\end{array}$ & UDAFF & UNAP & & \\
\hline $\begin{array}{l}\text { Respondieron la } \\
\text { encuesta }(\%)\end{array}$ & $29(6,9)$ & $36(8,7)$ & $38(9,2)$ & $312(75,2)$ & $23(6,7)$ & $33(9,6)$ & $36(10,5)$ & $252(73,2)$ & 759 \\
\hline $\begin{array}{l}\text { Seleccionados para } \\
\text { entrevista }(\%)\end{array}$ & $12(13,4)$ & $11(12,2)$ & $8(8,9)$ & $59(65,5)$ & $7(6,7)$ & $10(9,5)$ & $14(13,3)$ & $74(70,5)$ & 195 \\
\hline Entrevistados (\%) & $10(30,3)$ & $10(30,3)$ & $6(18,2)$ & $7(21,2)$ & $4(17,4)$ & $7(30,4)$ & $8(34,8)$ & $4(17,4)$ & 56 \\
\hline \multicolumn{10}{|l|}{$\begin{array}{l}\text { Estado civil de los padres } \\
\text { de los entrevistados (\%) }\end{array}$} \\
\hline Separados & $5(38,4)$ & $3(23,1)$ & $2(15,4)$ & $3(23,1)$ & $2(14,3)$ & $4(28,6)$ & $5(35,7)$ & $3(21,4)$ & 27 \\
\hline Casados & $2(18,1)$ & $4(36,4)$ & $1(9,1)$ & $4(36,4)$ & $1(20,0)$ & $2(40,0)$ & $1(20,0)$ & $1(20,0)$ & 16 \\
\hline Convivientes & $3(33,3)$ & $3(33,3)$ & $3(33,4)$ & 0 & $1(25,0)$ & $1(25,0)$ & $2(50,0)$ & 0 & 13 \\
\hline \multicolumn{10}{|l|}{$\begin{array}{l}\text { Nivel de educación de los } \\
\text { entrevistados (\%) }\end{array}$} \\
\hline Estudios secundarios & $8(72,7)$ & 0 & 0 & $3(27,3)$ & $3(60,0)$ & 0 & $2(40,0)$ & 0 & 16 \\
\hline Estudios técnicos & $2(40,0)$ & 0 & $3(60,0)$ & 0 & $1(33,3)$ & 0 & $2(66,7)$ & 0 & 8 \\
\hline Estudios universitarios & 0 & $10(58,8)$ & $3(17,7)$ & $4(23,5)$ & 0 & $7(46,6)$ & $4(26,7)$ & $4(26,7)$ & 32 \\
\hline
\end{tabular}

UDAFF: Universidad de Ayacucho Federico Froebel

UNAP: Universidad Nacional de la Amazonia Peruana en Loreto

C.S Mi Perú: Centro de Salud Mi Perú 
como el deseo sexual y la necesidad de experimentar (Figura 1).

Tanto varones como mujeres señalaron que su primera relación sexual fue en su casa o en casa de su pareja. Diversos participantes de ambos sexos sostuvieron que, en sus vínculos afectivos hay una priorización del aspecto sexual, por encima del aspecto sentimental o el compartir ideas u proyectos. Los hombres señalaron con frecuencia que su primera relación sexual fue con una pareja ocasional, mientras que las mujeres señalaron que fue con alguien que consideraban su "enamorado". Entre las razones que explican el inicio de relaciones sexuales (RS) en ambos géneros fue el consumo de alcohol. Las mujeres mencionaron que las razones por las cuales tuvieron RS fueron las presiones externas, tanto de la pareja definiéndolo como la "prueba del amor"; como por amigas que ya iniciaron su vida sexual y que suelen compartir sus experiencias sexuales, considerándose a sí mismas como "influenciadas" y con menor frecuencia por decisión propia, lo que incluye ilusión o por propios deseos de experimentar. En los varones primó más la propia iniciativa o decisión, alentado por lo que ellos llamaron curiosidad, o por "la pornografía que ven desde pequeños" y porque se consideraban en edad de tener relaciones sexuales; un grupo mayor mencionó que es lo esperable y valorado en su círculo social.

Las mujeres expresaron que luego del inicio sexual con su pareja ocurrieron cambios importantes en su relación, como mayor condescendencia y flexibilidad a los requerimientos del varón en demandas de "comportamiento" como en la forma de vestir y relación con los amigos, expresando sentimiento de dependencia. Ambos géneros señalaron los celos y las mujeres manifiestaron estar más abiertas al "perdón" en caso de infidelidad. Diversas mujeres también refirieron una mayor tendencia al maltrato verbal o físico por parte de sus parejas, ante la negativa a estos requerimientos. En relación a la duración del vínculo afectivo después del inicio sexual, las mujeres señalaron con más frecuencia que sus relaciones terminaron en breve tiempo después de esta experiencia.

En el mesosistema se incluye la relación del adolescente con su pareja y el uso de anticonceptivos como un tercer entorno, describiéndose las barreras individuales para su uso. Dentro de ello, las medidas de protección de preferencia a ser utilizados por los varones fue: el condón, la anticoncepción oral de emergencia (AOE) y el retiro. Las mujeres refirieron el uso de la $A O E$, el inyectable y la píldora. Sin embargo, ambos géneros reconocieron que el uso de estos anticonceptivos no es permanente. Un grupo de entrevistados priorizó su uso con parejas estables a la que conocen y en la que confían, y otros con parejas ocasionales o fortuitas (Figura 2).

El uso de la AOE se da en situaciones de no haber usado el condón, pero, principalmente, cuando el varón no quiere usarlo por cuestiones de sensibilidad o incomodidad. También se recurre al uso de AOE cuando se ha roto el condón y asumir que los contenidos seminales en contacto con los genitales o vagina podrían generar un embarazo.

\begin{tabular}{|c|c|c|c|}
\hline & & \multirow[b]{2}{*}{ Masculino } & \multirow[b]{2}{*}{ Femenino } \\
\hline & & & \\
\hline \multirow{5}{*}{ 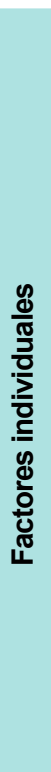 } & Primer enamorado & 12 - 14 años (más frecuente) & 12 - 14 años (más frecuente) \\
\hline & Inicio sexual & 14 - 15 años (más frecuente) & 15 - 16 años (más frecuente) \\
\hline & Perfil parejas sexuales & $\begin{array}{l}\text { Se suele tener con amigas... } \\
\text { mmm...más regaladas, se ve en la } \\
\text { forma de comportarse, en la forma } \\
\text { como se desenvuelve y habla.... } \\
\text { Lima, varón }\end{array}$ & $\begin{array}{l}\text { Mayormente hoy en día, con los } \\
\text { enamorados vemos más el tema sexual } \\
\text { que sentimental, ya no es compartir } \\
\text { ideas, conversas, intercambiar } \\
\text { opiniones. Ayacucho, mujer }\end{array}$ \\
\hline & $\begin{array}{l}\text { Efecto de la vida sexual } \\
\text { en la pareja }\end{array}$ & $\begin{array}{l}\text { Se comparte algo bonito con } \\
\text { una persona, pero puede llegar } \\
\text { hasta el punto de contagiarte } \\
\text { de una enfermedad o que salga } \\
\text { embarazada, y eso es mucha } \\
\text { responsabilidad. Ayacucho, varón }\end{array}$ & $\begin{array}{l}\text {...te aferras más a él porque lo amas y } \\
\text { confías y no lo quieres dejar, creía que } \\
\text { era para toda mi vida, a pesar de que } \\
\text { algunos hagan infinidad de cosas, esté } \\
\text { con otras chicas o te engañe, la mujer } \\
\text { igual sigue. lquitos, mujer }\end{array}$ \\
\hline & $\begin{array}{l}\text { Razones por las que } \\
\text { tienen relaciones } \\
\text { sexuales }\end{array}$ & $\begin{array}{l}\text { Por necesidad de conocerse uno al } \\
\text { otro, de experimentar y cuando la } \\
\text { chica te quiere y quiere hacerlo dice } \\
\text { que sí y acepta... Facebook, varón }\end{array}$ & $\begin{array}{l}\text { Si la relación es estable y tienen tiempo } \\
\text { y el chico no la ve, se disminuye el amor, } \\
\text { no se siente a gusto y se puede ir con } \\
\text { otra. Lima, mujer }\end{array}$ \\
\hline
\end{tabular}

Figura 1. Principales argumentos en la contextualización de la vida sexual del adolescente 


\begin{tabular}{|c|c|c|}
\hline \multirow{6}{*}{ 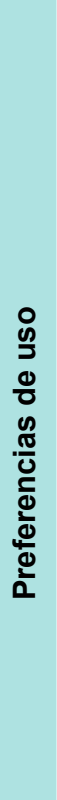 } & Masculino & Femenino \\
\hline & $\begin{array}{l}\text { Los hombres a veces usamos condón o la clásica } \\
\text { pastilla del día siguiente. Lima, varón }\end{array}$ & $\begin{array}{l}\text { Puedo usar la pastilla del dia siguiente si no quiere usar (la } \\
\text { pareja) condón. Lima, mujer }\end{array}$ \\
\hline & $\begin{array}{l}\text { No se siente igual. Se siente diferente pero mejor } \\
\text { es estar precavido, no?. lquitos, varón }\end{array}$ & $\begin{array}{l}\text { Mi enamorado no quería (condón) y al día siguiente o ese } \\
\text { mismo día me compraba la pastilla. lquitos, mujer }\end{array}$ \\
\hline & $\begin{array}{l}\text { Las mujeres quieren preservativo, pero, aprieta, se } \\
\text { siente menos, a veces ya pues...pero otras veces } \\
\text { yo digo que usamos la pastilla. Ayacucho varón }\end{array}$ & $\begin{array}{l}\text { La mayoría de las chicas que conozco usa la píldora del día } \\
\text { siguiente. Ayacucho, mujer }\end{array}$ \\
\hline & $\begin{array}{l}\text { Uso el preservativo, y si lo tengo sin preservativo la } \\
\text { pastilla del día siguiente. Facebook. varón }\end{array}$ & $\begin{array}{l}\text { Las pastillas usamos las chicas...y los hombres; los } \\
\text { preservativos, a veces. Facebook, mujer }\end{array}$ \\
\hline & $\begin{array}{l}\text { Si la chica no quiere en ese momento se deja de } \\
\text { hacerlo, pero el chico se amarga y se va a eso del } \\
\text { chongo o agarra con su ex porque las hormonas } \\
\text { todavía lo siguen. lquitos, varón }\end{array}$ & $\begin{array}{l}\text { A mi me paso una vez; el chico me dijo que si no lo hacemos; } \\
\text { [relaciones sexuales sin condón] terminamos. Yo, lo único que } \\
\text { hice fue levantarme e irme, porque si no me quiere cuidar, yo } \\
\text { me tengo que cuidar. Lima, mujer }\end{array}$ \\
\hline \multirow{3}{*}{ 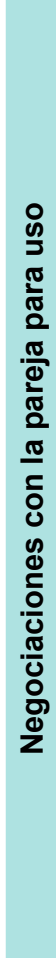 } & $\begin{array}{l}\text { Si la pastilla cuesta } 20 \text { son } 30 \text { soles más lo que voy } \\
\text { a ir a comprar más mi tiempo, el pasaje, el gasto } \\
\text { del hostal, pero si se va a un chongo [prostíbulo] } \\
\text { tienes relaciones con solamente } 20 \text { soles y te dan } \\
\text { tu presenvativo y faltando } 2 \text { minutos te tienes que } \\
\text { ir, si no te botan. Lima, varón }\end{array}$ & $\begin{array}{l}\text { Le pedí que use condón y ya no me siguió insistiendo que no } \\
\text { [su enamorado], el respeta lo que yo diga, aunque hay otros } \\
\text { que te reclaman, se molestan y te dicen que se siente mejor } \\
\text { sin condón; pero si en verdad te quisiera respetaría tu decisión. } \\
\text { lquitos, mujer. }\end{array}$ \\
\hline & $\begin{array}{l}\text { Siempre en esta edad tanto varones y mujeres } \\
\text { quieren experimentar al natural, y el varón exige, } \\
\text { y la mujer si está enamorada tiene que acceder. } \\
\text { Ayacucho, varón }\end{array}$ & $\begin{array}{l}\text { Bueno, en mi caso le dije [a su enamorado] que la pastilla del } \\
\text { día siguiente, es solo para una emergencia y que no me venga } \\
\text { a decir que no quiere cuidarse. Ha habidos casos donde me } \\
\text { decía que no quería usar condón, mejor que se busque otra } \\
\text { chica porque yo no quiero truncar mi futuro. Facebook, mujer }\end{array}$ \\
\hline & $\begin{array}{l}\text { Es mejor usar el choque y fuga porque tú no sabes } \\
\text { con qué hombre se ha metido, y también con la } \\
\text { ex, porque tú ya la has dejado. Con la estable tú } \\
\text { ya sabes cómo es ella, entonces hasta puedes } \\
\text { querer tener un hijo. Pero hay chicos que se ponen } \\
\text { tercos con la estable, que la chica quiere usar } \\
\text { preservativo y él no quiere porque es incómodo, } \\
\text { que mejor al natural. Facebook, varón }\end{array}$ & $\begin{array}{l}\text { Reconozco que las mujeres pueden decir que no [negarse a } \\
\text { tener relaciones sexuales sin condón] pero quieren hacerlo } \\
\text { y es mentira eso que dicen "me dejé llevar"; porque me ha } \\
\text { pasado. No es que te dejes llevar, es porque tú quieres hacerlo } \\
\text { y haces que el chico te lleve a propósito. Así es como ellos } \\
\text { quieren tener relaciones contigo, es como que esperas que sea } \\
\text { él quien te lo pida porque.... ósea, la mayoría de los hombres } \\
\text { tienen un pensamiento muy machista; ellos creen que si tú } \\
\text { eres la primera en decirles que quieres tener relaciones y que } \\
\text { usen condón; ellos van a decir que eres fácil y luego te dejan. } \\
\text { Ayacucho, mujer }\end{array}$ \\
\hline
\end{tabular}

Figura 2. Contexto del adolescente informado relacionado al uso de anticonceptivos, con enfoque por género.

La falta de uso de métodos de protección se relacionó a la limitada capacidad de negociación de la mujer, ya que varias de ellas mencionaron tener dificultad de comunicarse con claridad sobre sus deseos e inquietudes con sus parejas sexuales varones. Diversas entrevistadas compartieron que, al exigir el uso del condón, el varón con frecuencia se ofende, o sugiere emplear AOE o inyectables. Algunas entrevistadas mencionaron que su pareja puede maltratarlas en forma verbal, física y/o sexual. Para evitar esta situación las mujeres prefieren evitar exigir el uso de anticonceptivos y no utilizan ningún método de protección, asimismo, el varón solicita tener RS sin condón 
seduciéndola como parte de una prueba de amor, en caso de rehusarse, el varón puede terminar, suspender la relación afectiva y/o enojarse (Figura 2).

Los varones, sobre este aspecto, expresaron que las parejas casuales o fortuitas pueden exigirles usar condón, pero no las parejas estables, debido a que representa una señal de desconfianza hacia su salud y fidelidad. Además, refieren que las mujeres tienen temor de exigirles usar condón, resaltando que, finalmente, su uso depende solo de ellos. Algunos varones expresaron que, si la mujer carga un condón con ella, es porque está deseando RS, y que en caso de que una mujer no ceda a tener RS, el varón puede acudir a un prostíbulo.

En la Tabla 2 se presentan las causas por las que los entrevistados reportaron no usar anticonceptivos de acuerdo a la frecuencia con la que se presentan estos argumentos. La tabla permite observar la importancia de cada razón y como esta se asemeja o difiere según género.

Finalmente, los participantes resaltaron que en las ocasiones en las que tuvieron sexo sin protección tuvieron el riesgo de adquirir una enfermedad de transmisión sexual o VIH, lo cual detendría su proyecto de vida, y podría generar un embarazo no deseado, lo que truncaría su vida, por cuidar a un hijo de forma temprana, o podría terminar en un aborto (Figura 3).

\section{DISCUSIÓN}

El presente estudio identifica barreras que limitan la posibilidad de uso de anticonceptivos en adolescentes sexualmente activos e informados, con un enfoque por género en diferentes contextos geográficos peruanos. Se resalta la limitada capacidad de negociación en la mujer, el tipo de relación estable o fortuita con la pareja sexual, la presión del varón como pareja sexual, y el anticonceptivo de elección usado por el varón y por la mujer, siendo el condón y la $A O E$, respectivamente.

En relación a estos hallazgos, evidencia local ${ }^{(8)}$ e internacional ${ }^{(17)}$ sostiene que no existe relación significativa entre el conocimiento en salud sexual y la conducta sexual de riesgo ejercida por los adolescentes. Estos estudios resaltan que el ejercicio sexual ocasional sin anticonceptivos incrementa el riesgo de embarazo y de ETS ${ }^{(18)}$. El presente estudio profundiza en las razones que explican estos comportamientos en adolescentes, que cuentan con información según el tipo de relación afectiva de la pareja sexual. Esta situación contrasta con países africanos donde, a pesar del inicio temprano del ejercicio sexual y existiendo conciencia de la posibilidad de embarazo y riesgo de contraer ETS, los adolescentes tienen muy poco nivel de conocimiento sobre las formas de prevención ${ }^{(19)}$.

En concordancia con nuestros resultados, el estudio de Bayer et al. en 2011 y Flores et al. en 2012 manifiestan que la necesidad de afecto es crucial sobre todos los aspectos,

Tabla 2. Principales causas individuales para no usar anticonceptivos, según frecuencia de argumentos y por género

\begin{tabular}{|c|c|c|}
\hline \multirow{2}{*}{ Factores individuales identificados para no usar anticonceptivos } & \multicolumn{2}{|c|}{ Sexo } \\
\hline & Varón & Mujer \\
\hline Por las incomodidad del condón como raspaduras, alergias, menor sensibilidad y fácil rompimiento. & 1. ${ }^{\mathrm{a}}$ causa & 1. ${ }^{\mathrm{a}}$ causa \\
\hline Porque no saben exactamente cómo funciona el anticonceptivo sea ritmo, píldora o inyectable. & 7. ${ }^{\mathrm{a}}$ causa & 7. ${ }^{\mathrm{a}}$ causa \\
\hline $\begin{array}{l}\text { Por inaccesibilidad al condón durante el momento sexual íntimo, haciéndose difícil obtenerlo por cuestiones } \\
\text { de tiempo, lugar. }\end{array}$ & 3. ${ }^{a}$ causa & 2. ${ }^{a}$ causa \\
\hline Por vergüenza en ir a comprarlos y someterse a comentarios, observaciones o juicios. & 5. ${ }^{\mathrm{a}}$ causa & 4. ${ }^{\mathrm{a}}$ causa \\
\hline Porque creen que no va a pasar nada, ya que no ha pasado antes ni a ella o él ni a sus pares. & 6. ${ }^{\mathrm{a}}$ causa & 7. ${ }^{\text {a }}$ causa \\
\hline Porque pueden usar el retiro (coito interrumpido) & 6. ${ }^{\text {a }}$ causa & $5 .^{\text {a }}$ causa \\
\hline Porque algunas relaciones se dan con parejas fortuitas, de ocasión, no planeadas "choque y fuga". & 6. ${ }^{a}$ causa & 4. ${ }^{\mathrm{a}}$ causa \\
\hline Por tener una pareja estable, saludable, fiel, confiable a la que conocen. & 8. ${ }^{\mathrm{a}}$ causa & 3. ${ }^{\text {a }}$ causa \\
\hline Porque es más placentero hacerlo al natural. & 9. ${ }^{\text {a }}$ causa & 3. ${ }^{\mathrm{a}}$ causa \\
\hline Porque no le gusta usar anticonceptivos que puedan aumentar su peso. & 2. ${ }^{a}$ causa & NA \\
\hline $\begin{array}{l}\text { Por temor a incomodar a su pareja sexual y su reacción: Alejarse por un tiempo, terminar la relación u } \\
\text { ofenderlo al exigirle usar condón. }\end{array}$ & 4. ${ }^{\mathrm{a}}$ causa & NA \\
\hline Porque saben que después podrían usar el AOE. & 6. ${ }^{\mathrm{a}}$ causa & NA \\
\hline Por falta de dinero, por temor a ser encontrados por sus padres o familiares, por rebeldía hacia los padres. & 8. ${ }^{a}$ causa & NA \\
\hline Porque consideran que es rol de la mujer cuidarse y/o saber sus días de riesgo. & NA & $6 .^{a}$ causa \\
\hline Porque son inmaduros. & NA & 7. a causa \\
\hline
\end{tabular}

AOE: Anticonceptivo oral de emergencia

NA: No aplica, porque la pregunta estaba orientada al sexo opuesto 


\section{Masculino}

\section{Femenino}

..si sales embarazada ya no puedes seguir estudiando por tu bebe y no puedes cumplir las metas que te habías trazado. Lima, mujer
La primera puede ser un embarazo que repercute tanto a la chica como al chico y en cierto modo trunca el desarrollo de cada uno; el otro caso puede ser contagiarte de SIDA y marcar tu vida para siempre. Ayacucho, varón

Si sale embarazada y no sabes de quién, es porque estaba tomada o no era tu firme, no le van a tomar importancia y lo más probable es el aborto. Facebook, varón

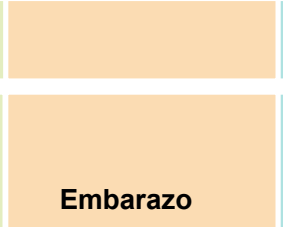

Embarazo

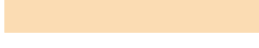

ETS
Varias tienen que abortar, a veces es la misma mamá quien te hace abortar, como el caso de mi hermana, pero antes ella (su mama) no estuvo apoyándola ni sabía lo que hacía (su hermana). Ayacucho, mujer

Figura 3. Consecuencias percibidas al no usar anticonceptivos por los adolescentes, según género

y representa un rol crítico en la sexualidad durante la adolescencia ${ }^{(20,21)}$. De esta manera, la capacidad de negociación en el uso coordinado de anticoncepción según el tipo de pareja sexual, es un aspecto relacionado en distintos grados a las consideraciones afectivas.

La evidencia regional sostiene que las intervenciones educativas deben incluir, además de información, habilidades sociales como asertividad, comunicación interpersonal, autoestima y otras herramientas que les permitan optimizar sus relaciones afectivas ${ }^{(22)}$. En relación al género, estudios latinos y locales sostienen que son los hombres los que presentan mayor información y prácticas sexuales de mayor riesgo en comparación a las mujeres ${ }^{(23,24)}$. Estudios en Cuba y España reportan, de manera similar, la tendencia de asignar la responsabilidad del uso de anticonceptivo a la mujer, así como la creencia de los varones de sentirse con derecho de exigir a su pareja tener $\mathrm{RS}^{(25-26)}$.

Un estudio desarrollado por la Organización Mundial de la Salud en el Perú, concluye que, si bien el Programa Nacional de Educación Sexual tiene potencial para fortalecer la salud sexual y reproductiva desde la pubertad, en la práctica, las metas aún representan un desafío, sugiriendo que, si valoraran en sus contenidos aspectos culturales, como la desigualdad de género según los presentes hallazgos, este programa podría responder a la información que nuestros adolescentes quieren y necesitan ${ }^{(27)}$.

Dada la naturaleza sensible de estos temas, existe la posibilidad de que los participantes del presente estudio hayan generado sesgo de información, es decir, aceptabilidad social en la respuesta a algunas preguntas, buscando responder de acuerdo a normas sociales o sexuales propias de la edad, localidad o por género. Con el objetivo de prevenir este sesgo, el presente estudio utilizó instrumentos diferenciados en género a través del refinamiento previo y constante de los instrumentos con adolescentes. Los hallazgos de este estudio no pueden ser extrapolados a otras poblaciones o a todo el Perú; sin embargo, por haberse realizado en tres importantes zonas geográficas del país en donde la tasa de EA es alta, permite transferir estos hallazgos a otros escenarios similares.

Hubiera sido ideal incluir en el presente estudio a menores de 18 años, pero se consideró que conversar sobre aspectos de la vida sexual activa en entrevistas podría llevar a testimonios de relevancia legal como por ejemplo abuso sexual, lo cual, podría llevar a romper el código de confidencialidad y privacidad ofrecido al participante para denunciar este delito. Por otro lado, pedir el asentimiento informado a los padres, hubiera implicado informarles, que sus hijos fueron seleccionados por haber iniciado su vida sexual y no usar anticonceptivos, rompiendo la confidencialidad ofrecida.

Un importante hallazgo de este estudio, es haber identificado oportunidades de desarrollar esta investigación en el componente virtual, demostrando que, en temas sensibles como la sexualidad, las redes sociales representan un escenario accesible, de amplia cobertura y útil en el abordaje de actividades preventivas, el cual podría ser explorado en futuros estudios. Existen reportes en otros contextos, que demuestran la influencia que tienen las redes sociales, las 
páginas pornográficas y demás plataformas virtuales en la información y conducta sexual del adolescente, refiriéndolas como una fuente de aprendizaje.

Además, se ha constatado que en el componente virtual, la distancia, la accesibilidad, la amplia cobertura y la familiaridad que tienen los adolescentes con esta vía, parecen ser ventajas importantes para un adecuado abordaje de diversas actividades preventivas promocionales, el cual podría ser considerado en futuras intervenciones, siempre que se manejen de manera segura y controlada, en el sentido de a quienes va dirigido, qué contenidos se difunden, etc. Diversos estudios asocian el uso de internet y las redes sociales a una mayor interacción y colaboración entre los usuarios, concluyendo que las dimensiones tecnológicas culturales y del mensaje son las que hacen la diferencia en el uso de estas herramientas en la comunicación en salud sexual y reproductiva ${ }^{(28)}$

Ampliando este panorama de uso, la adolescencia se desarrolla en un ecosistema muy ligado a los medios de comunicación, el tiempo que pasan frente a una pantalla es cada vez mayor. En el caso de localidades en vías de desarrollo, la literatura reporta que las revistas, los programas juveniles, los blogs son muy populares entre adolescentes ${ }^{(29)}$. El buscar información sobre aspectos de salud, personales o académicos son las principales razones por las cuales, los y las adolescentes emplean internet, representando una plataforma oportuna para el abordaje de la sexualidad. Estas evidencias en conjunto refuerzan la importancia que podrían representar estos medios en la difusión asertiva de habilidades para la vida.
Finalmente estos hallazgos podrían constituir una referencia en la optimización de las actuales intervenciones multisectoriales que promueven la educación sexual integral a través de información educativa, ya que, además, se podrían fortalecer los aspectos relacionados con la toma de decisiones como la capacidad de negociación o la tolerancia a la frustración, los cuales, además de ser importantes en el momento que la persona decida libremente si emplea o no un anticonceptivo, o si inicia su vida sexual, representan aspectos útiles que se extrapolan a distintos escenarios de su vida, aspirando a un bienestar integral.

Agradecimientos: a los adolescentes por su participación y apoyo en las distintas fases del estudio; a la socióloga Nina VanDer Styuff, a la Estrategia Nacional de Salud sexual y Reproductiva; a la Dirección Regional de Salud Loreto; y a los representantes de la Universidad de Ayacucho Federico Froebel (Cristhian Aldana), a la Dra. Gracia Subiría del Fondo Poblacional de las Naciones Unidas en Perú, y al comunicador Víctor La Noire.

Contribuciones de autoría: GC, JB y MC participaron en la concepción del estudio. GC, JB, SC participaron en la ejecución del proyecto, análisis e interpretación de los datos. GC escribió la primera versión del manuscrito. GC, JB, MC, MP y SC aportaron a la redacción y revisión crítica del contenido intelectual, todos aprobaron la versión final.

Fuentes de financiamiento: estudio financiado por el Instituto Nacional de Salud de Perú.

Conflictos de interés: los autores no presentan conflicto de interés en la publicación de este artículo.

\section{REFERENCIAS BIBLIOGRÁFICAS}

1. Horoszowski M. The Full List of the 17 United Nations Sustainable Development Goals [Internet]. MovingWorlds Blog. 2015 [citado 16 agosto 2016]. Disponible en: http://blog.movingworlds.org/the-fulllist-of-the-17-united-nations-sustainabledevelopment-goals-with-pictures-sdgs

2. Instituto Nacional de Estadística e Informática. Perú: Encuesta Demográfica y de Salud Familiar, 2014 [Internet]. Lima: Instituto Nacional de Estadística e Informática; 2015 [citado 15 octubre 2016]. Disponible en: https://www. inei.gob.pe/media/MenuRecursivo/ publicaciones_digitales/Est/Lib1211/ pdf/Libro.pdf

3. Centro Nacional de Epidemiologia, Prevención y Control de Enfermedades. Análisis de la Situación Epidemiológica del VIH/SIDA en el Perú, 2017 [Internet]. Lima: Ministerio de Salud: Dirección
General de Epidemiología; 2017. [citado 1 septiembre 2017]. Disponible en: http:// www.dge.gob.pe/portal/docs/vigilancia/ sala/2017/SE23/vih.pdf

4. Kostrzewa K. The sexual and reproductive health of young people in Latin America: evidence from WHO case studies. Salud Pública Méx. 2008;50(1):10-6.

5. DEVIDA. II Estudio Nacional: Prevención y consumo de drogas en estudiantes de secundaria - 2007 [Internet]. Lima: Gerencia de Prevención y Rehabilitación del Consumo de Drogas, Comisión Nacional para el Desarrollo y Vida sin Drogas; 2009. [citado 1 septiembre 2017]. Disponible en: http:// www.devida.gob.pe/wp-content/uploads/2014/12/II-Estudio-Nacional-Prevenci\%C3\%B3n-Y-Consumo-De-Drogas-En-Estudiantes-De-Secundaria_Resultados-Nacionales_-2007.pdf
6. Decreto Supremo que aprueba el Plan multisectorial para la Prevención del Embarazo en Adolescentes y constituye la Comisión Multisectorial Permanente encargada del seguimiento, monitoreo y evaluación del plan. Decreto supremo N012-2013-S del 7 de noviembre del 2013. El Peruano n ${ }^{\circ}$ 506616, (7-11-2013). Disponible en: ftp://ftp2.minsa.gob.pe/ normaslegales/2013/DS012_2013_SA_ EP_c.pdf

7. Cacéres CF, Mendoza W, Konda K, Lescano A. Nuevas evidencias para las políticas y programas de salud en VIH/SIDA e infecciones de transmisión sexual en el Perú: información disponible hasta febrero 2007. Lima: Universidad Peruana Cayetano Heredia; Organización Panamericana de la Salud; 2007. [citado 30 diciembre 2016]. Disponible en: http://www.bdigital.unal. edu.co/47336/1/9789972222146.pdf 
8. Jerónimo Maturana CA, Alvarez Leon JG, Carbonel Luyo WF, Neira Goyeneche J. Sexualidad y métodos anticonceptivos en estudiantes de educación secundaria. Acta méd peruana. 2009;26(3):175-9.

9. Chirinos JL, Brindis CD, Salazar VC, Bardales OT, Reátegui LR. Perfil de las estudiantes adolescentes sexualmente activas en colegios secundarios de Lima, Perú. Rev Med Hered. 1999;10(2):49-61.

10. Arriagada Barrera S. Adolescencia y acceso a salud reproductiva y educación sexual ¿Qué puede cambiar? [Internet]. Lima: Centro de Promoción y Defensa de los Derechos Sexuales y Reproductivos PROMSEX;2011. [citado9denoviembre 2015]. Disponible en: http://promsex. org/images/docs/Publicaciones/ adolescentesSaludreproductiva.pdf

11. Garcia PJ, Cotrina A, Cárcamo C. SEXO, PREVENCIÓN Y RIESGO. Adolescentes y sus madres frente al VIH y las ITS en el Perú. [Internet]. Lima: Fondo de Población de las Naciones Unidas; 2008. [citado 9 de noviembre 2015]. Disponible en: http://www.unfpa.org.pe/ publicaciones/publicacionesperu/CARESexo-Prevencion-Riesgo.pdf

12. UCSF Division of Prevention Science [Internet]. San Francisco: UC San Francisco; 2016 [acceso 1 septiembre 2016]. Survey Instruments and Scales. Disponible en: http://caps.ucsf.edu/resources/survey-instruments

13. Martins LB, Costa-Paiva L, Osis MJ, Sousa MH, Pinto Neto AM, Tadini V. Knowledge of contraceptive methods among adolescent students. Rev Saude Publica. 2006;40(1):57-64.

14. Ritchie J, Lewis J. Qualitative Research Practice: A Guide for Social Science Students and Researchers. London: SAGE Publications; 2003.356 p.

15. Netemeyer RG, Bearden WO, Sharma S. Scaling Procedures: Issues and Applica- tions. London: SAGE Publications; 2003. $225 \mathrm{p}$.

16. Bronfenbrenner U, Morris PA. The Bioecological Model of Human Development. En: Lerner RD, editor. Handbook of Child Psychology. New Jersey: John Wiley \& Sons, Inc.; 2007.

17. Trejo-Franco J, Flores-Padilla L, Villaseñor-Farias M. Percepción de riesgo de contraer SIDA en adolescentes de Guadalajara Jalisco. Rev Enferm Inst Mex Seguro Soc. 2010;18(1):3-8

18. Silva-Fhon J, Andrade-Iraola K, Palacios-Vallejos E, Rojas-Huayta V, Mendonça-Júnior J. Nivel de conocimientos y comportamientos de riesgo relacionados con infecciones de transmisión sexual entre adolescentes mujeres. Enferm Univ. 2014 Oct;11(4):139-44.

19. Bankole A, Biddlecom A, Guiella G, Singh S, Zulu E. Sexual Behavior, Knowledge and Information Sources of Very Young Adolescents in Four Sub-Saharan African Countries. Afr J Reprod Health. 2007;11(3):28-43.

20. Juarez F, Bayer AM. "Without a plan" but "keeping on track": views on contraception, pregnancy and abortion in Mexico City. Glob Public Health. 2011;6 Suppl 1:S90110. doi: 10.1080/17441692.2011.581674.

21. Flores Tejada ME. Conocimientos, prácticas sexuales y actitud del adolescente hacia la sexualidad responsable y embarazo precoz [Tesis]. Lima: Universidad Ricardo Palma; 2012 [citado 19 agosto 2016 ]. Disponible en: http://cybertesis.urp.edu. pe/handle/urp/287

22. Moreno S, Leon Canelón M, Becerra L. Conducta sexual, conocimiento sobre embarazo y necesidades percibidas con relación a educación sexual, en adolescentes escolarizados. Espacio Abierto. 2006; 15(4):787-803.
23. Martínez J, Parada LY, Duarte ZK . Prevalencia de uso de condón en todas las relaciones sexuales con penetración durante los últimos doce meses y factores asociados en estudiantes universitarios entre 18 y 26 años de edad. Rev Univ salud. 2014;16(2):198- 206.

24. Soto V. Factores asociados al no uso del condón. Estudio en adolescentes y adultos jóvenes de Chiclayo. An Fac med. 2006;67(2):152-9.

25. Guerrero Masiá MD, Guerrero Masiá M, García-Jiménez E, Moreno López A. Conocimiento de los adolescentes sobre salud sexual en tres institutos de educación secundaria valencianos. Rev Pediatr Aten Primaria. 2008;10:433-42.

26. González JC. Conocimientos, Actitudes y Prácticas sobre la Sexualidad en una Población Adolescente Escolar. Rev salud pública. 2009;11(1): 14-26.

27. Cookingham LM, Ryan GL. The impact of social media on the sexual and social wellness of adolescents. J Pediatr Adolesc Gynecol. 2015;28(1):2-5. doi: 10.1016/j. jpag.2014.03.001.

28. Bacallao-Pino LM. Recursos interactivos y redes sociales en la información sobre salud sexual y reproductiva en la prensa cubana: la sección "Sexo sentido", del diario Juventud Rebelde. Rev Cuba InfEn Cienc Salud. 2015;26(2): 94-106.

29. Borzekowski DL, Rickert VI. Adolescent cybersurfing for health information: a new resource that crosses barriers. Arch Pediatr Adolesc Med. 2011;155(7):813-7.

\footnotetext{
Correspondencia: Gloria Carmona

Dirección: Calle Santa Felicidad 222 Pando 3ra etapa. Cercado de Lima, Perú

Telefono: (+51) 7480000

Correo electronico: gloriacarmona.c@gmail.com
}

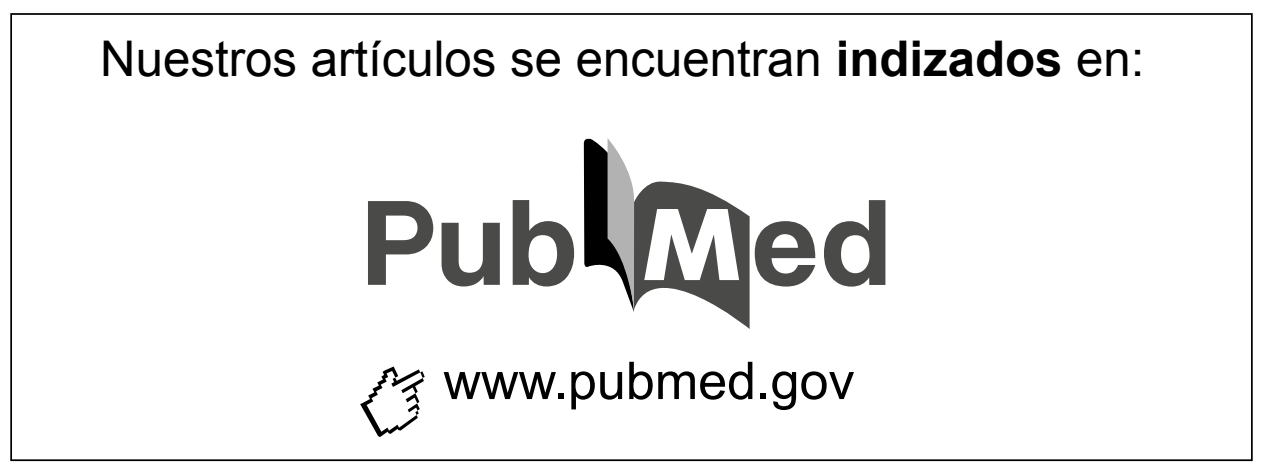

